\title{
Multilinguales
}

\section{L'ethos collectif des altermondialistes dans les forums sociaux mondiaux}

The collective ethos of altermondialists in world social forums

\section{Azzedine Kadir}

\section{(2) OpenEdition}

\section{Journals}

\section{Édition électronique}

URL : https://journals.openedition.org/multilinguales/5165

DOI : $10.4000 /$ multilinguales. 5165

ISSN : 2335-1853

\section{Éditeur}

Université Abderrahmane Mira - Bejaia

\section{Référence électronique}

Azzedine Kadir, "L'ethos collectif des altermondialistes dans les forums sociaux mondiaux », Multilinguales [En ligne], 13 | 2020, mis en ligne le 15 juillet 2020, consulté le 18 mai 2021. URL : http:// journals.openedition.org/multilinguales/5165; DOI : https://doi.org/10.4000/multilinguales.5165

Ce document a été généré automatiquement le 18 mai 2021

\section{cc)}

Multilinguales est mise à disposition selon les termes de la Licence Creative Commons Attribution Pas d'Utilisation Commerciale - Pas de Modification 4.0 International 


\section{L'ethos collectif des altermondialistes dans les forums sociaux mondiaux}

The collective ethos of altermondialists in world social forums

Azzedine Kadir

1 Le discours des organisations altermondialistes est un phénomène peu étudié en analyse de discours. Le mouvement fait partie de la constellation des formes de mobilisation citoyenne que semblent aujourd'hui préférer les altermondialistes qui délaissent de plus en plus les formes de lutte traditionnelles. Nés vers la fin des années quatre-vingt-dix, les forums sociaux mondiaux (désormais FSM) sont aujourd'hui répandus dans le monde entier, notamment aux USA, dans les pays d'Amérique du Sud et en Afrique. Notre étude est née d'une suite de questions: quelle image les altermondialistes projettent-ils dans les FSM ? À quoi est dû cet ethos collectif? Comment les altermondialistes réagissent-ils au discours dominant sur mondialisation? Pour répondre à ces questions, notre article va donc analyser la construction de l'image de soi qui s'est effectuée dans le discours altermondialiste lors des différents FSM et la façon dont elle s'est mise en place à travers une polarisation entre mondialisation et altermondialisation. En raison de la visée argumentative de cette construction, nous recourons à la notion d'ethos dans son sens rhétorique, à savoir l'image de soi que le locuteur construit à travers son discours pour exercer une influence sur son auditoire. Et comme il s'agit d'une construction collective qui concerne un groupe, un ensemble d'intervenants et d'individus concernés, nous choisirons de la dénommer « ethos collectif ».

\section{Cadrage théorique}

2 La notion d'ethos a été abordée plusieurs fois par l'analyse du discours et la théorie de l'argumentation. Nous retiendrons ici la définition qu'en donne R. Amossy: "l'image [de l'orateur] construite dans le discours » (Amossy, 1999 : 154). C'est en effet par des 
stratégies discursives de construction de l'identité collective et de polarisation entre mondialisation et altermondialisation que l'identité du groupe se construit. Nous retenons la définition suivante d'Orkibi concernant l'ethos collectif :

«Image de soi d'un groupe: une image mobilisée ou reflétée dans la parole de l'individu appartenant à ce groupe [...] mais aussi une image de soi collective qui est exprimée par un groupe, à savoir l'ethos du locuteur collectif » (Orkibi, 2012 : 22)

Or, il semble particulièrement pertinent dans notre cas, d'intégrer aussi la notion élaborée par P. Charaudeau d'un ethos collectif hétéro-attribué, à savoir la projection sur un groupe d'une image sociale identitaire, artificiellement homogène, fondée sur des aprioris et des stéréotypes, de la part de ceux qui ne lui appartiennent pas :

«L'ethos collectif correspond à une vision globale, mais à la différence de l'ethos singulier, il n'est construit que par attribution apriorique, attribution d'une identité émanant d'une opinion collective vis-à-vis d'un groupe autre. » (Charaudeau, 2005 : 90)

Charaudeau envisage ici l'ethos comme une construction extérieure à la source énonciative qui le porte. Il semble par ailleurs utile, dans le contexte du mouvement altermondialiste, d'intégrer les propos de Declercq qui souligne ce point :

"Tout ce qui, dans l'énonciation discursive, contribue à émettre une image de l'orateur à destination de l'auditoire. Ton de voix, débit de la parole, choix des mots et arguments, gestes, mimiques, regard, posture, parure, etc., sont autant de signes, élocutoires et oratoires, vestimentaires et symboliques, par lesquels l'orateur donne de lui-même une image psychologique et sociologique » (Declerc, $1983: 48$ )

Cette double rhétorique d'identification et de polarisation produit une image du groupe avec laquelle les membres du mouvement peuvent s'identifier et à l'aide de laquelle ils se positionnent par rapport aux autres groupes. L'étude de l'ethos hétéro-attribuéd'un groupe exige d'analyser la manière dont ce groupe élabore sa propre image et " retravaille une série de représentations sociales préétablies. » (Orkibi, $2008: 4$ )

Il est également bien connu que l'autre discours produit sur la mondialisation constitue une composante identitaire fondamentale pour la communauté des altermondialistes. Non seulement un discours porte avec lui une image de soi plus ou moins valorisante ou valorisée, mais chaque locuteur doit, dans le contexte de la mondialisation, se positionner par rapport au discours dominant sur la mondialisation: d'abord, par la prise de position qui se révèle dans le discours altermondialiste, ensuite identifier qui parle, qu'est-ce qui est dit et enfin comment on le dit. C'est précisément par la narration de l'histoire du mouvement, par son positionnement dans l'espace public mondial et par la description de ses liens de filiation que l'identité des organisations altermondialistes se dessine.

\section{Corpus et méthodologie}

Cet article a comme principal objectif d'analyser le discours tenu par des acteurs sociaux (écologistes, militants, économistes) entourant la question de la mondialisation à la fin des années 1990, tel qu'il se présente dans les textes altermondialistes. Cette analyse suppose une catégorisation de leurs positionnements à partir d'unités de classification, telles que les définitions, les énoncés explicatifs, les énoncés modalisés, les marqueurs d'opposition, etc. Nous chercherons ainsi à voir comment les définitions sont formulées (dans la prise en compte de l'autre, par exemple), qui les énonce, quelles positions sociales sont revendiquées, etc., afin d'en faire ressortir l'argumentation. 
Concernant la méthodologie et l'analyse des données, nous allons d'abord présenter celles qui concernent les textes sur le mouvement altermondialiste, dans lesquels nous utiliserons la notion d'ethos hétéro-attribué. Pour les fins de notre analyse, nous avons retenu les textes qui traitent de la mondialisation à partir de 2001, date de la première édition du FSM organisé à Porto Alegre (Brésil).

7 Notre corpus se compose d'un ensemble de textes numériques (déclarations, forums, pages web, etc.) identifiés sur le site de l'organisation altermondialiste appelée Comité pour l'annulation de la dette du Tiers Monde (désormais CADTM). Cette organisation s'est déclenchée principalement en Belgique. Depuis sa création en 1990, cette organisation altermondialiste ancre son action au carrefour des luttes des mouvements sociaux populaires, des syndicats, des comités de solidarité internationale et des ONG de développement. Membre du conseil international du FSM, le CADTM s'inscrit dans le mouvement citoyen international qui lutte pour d'autres mondes possibles, ébauchant les traits d'une mondialisation alternative à celle que les promoteurs du modèle capitaliste, néolibéral et globalisé veulent imposer aux peuples. Nous avons effectué une recherche de l'expression « mondialisation » et de ses antonymes (ce qui constitue le champ discursif de l'altermondialisation). Au total, nous avons assemblé 20 textes consacrés à l'altermondialisation rédigés et lus lors des différents FSM.

Ces textes sont hétérogènes à plusieurs titres :

- Les énonciateurs, les destinataires et les buts: des énonciateurs et des destinataires différents, ainsi que des buts différents (argumenter, convaincre, séduire, inciter à l'action) donnent lieu à des stratégies de construction de l'ethos et de l'identité très différentes.

- Le thème : la problématique de la mondialisation, par exemple, fait l'objet d'un traitement différent selon qu'elle est appréhendée par tel ou tel énonciateur.

- Corpus activistes: Les textes utilisés dans notre travail sont désignés uniquement par le titre, le nom de l'auteur, l'année de l'énonciation et le nom de l'organisation ou de l'institution.

\section{Ethos et choix des arguments}

8 L'ethos montré que projettent les altermondialistes est élaboré par le moyen du choix des arguments et des procédés qu'ils utilisent pour se défendre et en même temps pour travailler leur ethos collectif. Il existe également un autre type d'argumentation dont l'usage est très récurrent dans les FSM, à savoir l'argument d'autorité qui consiste à faire admettre une thèse en la rapportant à son auteur considéré comme source d'autorité et digne de foi. C'est le cas de la Déclaration Universelle des Droits de l'Homme et la Charte des Nations-Unies que les altermondialistes utilisent fréquemment dans les FSM afin de permettre au discours altermondialiste de justifier son argumentation. En effet, dans les textes altermondialistes, le choix des arguments renvoie à deux phénomènes.

\subsection{La question de l'argument d'autorité : faire référence}

9 Faire référence à l'argument d'autorité constitue un procédé important dans la construction de l'ethos collectif altermondialiste. Dans les énoncés de notre corpus, par exemple, les nombreuses références aux chartes et aux différents traités internationaux consolident la démonstration : 
«Le raisonnement par autorité constitue un type de démonstration parmi d'autres et il faudrait le cataloguer à côté du raisonnement par récurrence de l'induction ou $\mathrm{du}$ raisonnement par analogie dans un inventaire des types de preuves reconnues comme valides à telle époque, dans telle collectivité intellectuelle. » (Ducrot, 1984 : p. 167)

10 Par ailleurs, l'argument d'autorité élabore une légitimation du discours altermondialiste. Il montre que les textes altermondialistes sont le fruit de travaux et de réflexions et surtout qu'ils sont réalisés à la demande des instances internationales. Ils sont débattus au sein d'une communauté d'experts ce qui leur attribuerait une valeur de légitimité. En fait, l'une des caractéristiques du discours altermondialiste est de se légitimer en altérant le discours dominant sur la mondialisation. Les énoncés suivants, prélevés dans les énoncés du corpus, illustrent ce fait discursif :

(1) «La riposte possible de l'altermondialisme et des multiples acteurs qui résistent à la spoliation néolibérale doit les conduire à articuler leurs actions et à se mobiliser autour d'objectifs communs, qui peuvent être présentés comme des propositions visant à l'élaboration d'un nouveau Contrat Social, une nouvelle Charte des Droits, à caractère mondial, à l'image de la Déclaration Universelle des Droits de l'Homme, de 1948. » (Texte 19)

(2) «La Déclaration Universelle est universelle, elle lie les États qui l'ont ratifié dans leurs actions spécifiques et dans leurs responsabilités en tant qu'actionnaires ou que membres des institutions internationales. » (Texte 8)

(3) «La Charte devrait être rediscutée autour de la Déclaration Universelle des Droits de l'Homme qui fonde la légitimité des Nations Unies et sur le rôle de l'ONU dont l'objectif devrait être d'aider à la transformation du monde plutôt que d'œuvrer à sa reproduction. » (Texte 14)

(4) «Comme nous l'avons affirmé au départ, le fondement du droit international, ne peut être que la Déclaration Universelle des Droits de l'Homme. Les Nations Unies peuvent s'ouvrir à la complexité des sociétés, reconnaître les acteurs émergents, leur donner une légitimité. » (Texte 6)

11 Les énoncés (1), (2), (3) et (4) convergent vers une seule et même conclusion dans leur argumentation, à savoir que tout se fait en relation avec la Déclaration Universelle des Droits de l'Homme. Les FSM coordonnent des actions de dimension internationale afin d'aider à la transformation du monde plutôt que d'œuvrer à sa reproduction. L'orientation argumentative du discours altermondialiste insiste fortement sur la légitimité et le rôle primordial des Droits de l'Homme et des citoyens dans le contexte de la mondialisation. Une autre raison peut expliquer cet empressement à mettre en avant l'impact du discours altermondialiste qui induit un approfondissement de la conception de la citoyenneté et du rôle des citoyens dans la garantie des droits. Mais cette conception de la citoyenneté n'est pas en fait partagée par tous les États.

L'enjeu essentiel du discours altermondialiste est d'articuler arguments et contrearguments afin de lui donner un caractère ouvert et des valeurs partagées au niveau mondial, en s'appuyant sur des discours argumentatifs élaborés et acceptés par tous :

« Il s'agit d'un discours élaboré au sens où il correspond à un discours tel qu'on peut l'attendre dans sa forme adulte : présence d'arguments susceptibles d'être acceptés car faisant appel aux valeurs d'une culture partagée par le locuteur et l'interlocuteur, et de contre-arguments (ou de toute autre forme de négociation indiquant que le locuteur prend en compte les arguments qu'on pourrait lui opposer). » (Golder, $1996: 120)$ 
13 L'extrait ci-dessus rappelle, à ce propos, clairement les enjeux qui se dessinent et éclairent les raisons d'un tel procédé discursif qui légitime en permanence le discours altermondialiste par des arguments d'autorité élaborés. L'énonciation de la Déclaration Universelle des Droits de l'Homme servant comme argument d'autorité n'est pas le seul procédé discursif utilisé dans les altermondialistes. Les nombreux énoncés analysés font également renvoi à de multiples forums sociaux tenus dans différents pays ou à des déclarations rédigées et lues à la fin de chaque forum. L'objectif reste toujours le même, celui de la légitimité discursive.

14 Pour légitimer le discours altermondialiste, il est nécessaire de l'énoncer dans le discours lui-même en y faisant référence. Les deux énoncés suivants attestent des arguments avancés :

(5) «Le Forum Social Mondial de Porto Alegre a confirmé les orientations de la rencontre européenne du 6 janvier 2000. Il a permis de les situer dans une nouvelle perspective, celle d'une nouvelle phase des mobilisations sur la mondialisation. » (Texte 3)

(6) «L'orientation qui se dégage des Forums Sociaux pour passer des résistances aux alternatives met en avant l'accès aux droits pour tous. La réponse est que la modernisation la plus intéressante est celle du respect, de la garantie et de l'approfondissement des droits fondamentaux. » (Texte 8)

15 Les énoncés (5) et (6) font une référence directe aux FSM avec l'indication du lieu et de la date. Ce procédé identifie le discours altermondialiste comme appartenant à un espace dynamique puisque c'est un moyen d'interaction entre les différentes organisations altermondialistes. À ce sujet, la Charte de principes du forum social mondial évoque, dans son article 8 , ce procédé propre au discours altermondialiste :

«Le Forum Social Mondial est un espace pluriel et diversifié, non confessionnel, non gouvernemental et non partisan, qui articule de façon décentralisée, en réseau, des instances et mouvements engagés dans des actions concrètes, au niveau local ou international, visant à bâtir un autre monde. »

16 Cette Charte de principes recherche, en effet, un ancrage social aux valeurs que le discours altermondialiste défend dans la mesure où il invoque un caractère universel de ces valeurs. Les renvois aux FSM permettent aussi d'orienter le discours altermondialiste vers une légitimité plus grande sur un ensemble de propos que les leaders ou les simples militants tiennent sur eux-mêmes, sur leurs actions, leurs thèses, leurs réussites et leurs échecs. Un autre argument peut également être avancé pour expliquer les raisons de cet aspect auto-référentiel permanent. La construction du discours altermondialiste correspondrait à une contestation forte où les altermondialistes auraient identifié la mondialisation comme étant un adversaire qui vise à rationnaliser un monde globalisé, en reléguant au second plan la légitimité de la souveraineté et de la liberté d'action des États et des gouvernements. Ces derniers, seraient dès lors limités à ne faire que ce que leur imposent les institutions internationales.

La référence permanente aux FSM indique la bonne conformité des promoteurs de l'altermondialisation à cet imaginaire particulier d'un autre monde possible et de son changement. Par ce renvoi à de multiples forums sociaux, on retrouve à nouveau le poids du discours altermondialiste dans la construction de sa légitimité tant critiquée faute de pouvoir. 


\subsection{Le forum des activistes : un ethos collectif recherché par les altermondialistes}

« Nous devons être capables de faire régulièrement la démonstration du caractère massif de nos initiatives. » (Texte 1)

« Nous devrons nous appuyer beaucoup plus sur le travail et les réseaux militants.» (Texte 4)

" Nous devons rester totalement ouverts à toutes ces initiatives en marche. » (Texte 5)

«À nous tous de construire cette revendication, ce à quoi nous œuvrons lors de nos manifestations. » (Texte 9)

« Nous rentrons dans une crise profonde et longue. » (Texte 11)

« Nous défendons des propositions qui doivent d'abord appuyer la prise de conscience la plus large. » (Texte 15)

« Nous nous battons aujourd'hui pour lutter contre la mondialisation néolibérale.» (Texte 8)

« Nous sommes engagés dans toutes les luttes de résistance. » (Texte 12)

Les acceptions du pronom «nous » relèvent essentiellement de deux sens : le premier réfère à une valeur générale : « À nous tous de construire cette revendication, ce à quoi nous œuvrons lors de nos manifestations. » et réfère à tous les citoyens du monde (l'ensemble de l'humanité) y compris les rédacteurs et les signataires des déclarations lors des FSM. Le deuxième réfère à un groupe spécifique plus particulièrement les militants altermondialistes :

" Nous devrons nous appuyer beaucoup plus sur le travail et les réseaux militants » « Nous sommes engagés dans toutes les luttes de résistance »

Multilinguales, 13 | 2020 
« Nous devons être capables de faire régulièrement la démonstration du caractère massif de nos initiatives»

21 Les énoncés ci-dessus montrent l'implication des altermondialistes dans le procès, et généralisent leurs propos à un groupe dont ils font partie, afin de créer une communion entre ses militants dans le but de favoriser leur adhésion à une même collectivité. Cet emploi permet à un locuteur de se construire une identité altermondialiste, symbole d'une appartenance à une communauté de valeurs, pour être reconnu et accepté en tant que membre de cette communauté. Par l'emploi du pronom "nous » les locuteurs construisent et expriment leur identité altermondialiste dans le but de rassembler les citoyens derrière eux. On peut ainsi supposer que l'argumentation est tournée principalement vers l'humanité : les citoyens du monde.

L'étude du pronom personnel "nous " permet d'apporter une première conclusion sur le fonctionnement du discours altermondialiste de CADTM. Le discours altermondialiste dispose d'une large palette de formulations qui l'autorise à contredire les orientations argumentatives du discours dominant sur la mondialisation et il se décline sur les modes de la contre-argumentation et de l'exhortation. Le discours altermondialiste peut être aussi placé sur une échelle de valeurs permettant d'évaluer la portée de chacun de ses énoncés et ainsi d'enrichir les contre arguments selon les besoins du contexte. Enfin, une remarque s'impose : l'analyse a permis de confirmer le fait que les éléments constitutifs du discours altermondialiste interagissent et qu'il n'est pas rare de voir une richesse de combinaisons variées entre le pronom « nous » et ses référents.

\subsection{Nous et eux : axiologisation et stratégies de polarisation}

L'axiologisation concerne le processus d'attribution de valeurs modales à la signification des mots. Elle renvoie aux systèmes de valeurs et à la connaissance du monde par les locuteurs fonctionnant sur des universaux sémantiques.

«Nous appelons axiologiques les unités linguistiques qui reflètent un jugement d'appréciation, ou de dépréciation, porté sur le sujet dénoté, par le sujet d'énonciation. » (Kerbrat-Orecchioni, 1977 : 110)

En effet, un jugement de valeur porté sur des mots peut fonctionner comme un critère permettant de construire certaines catégories d'objets. Donc nous pouvons affirmer que les jugements de valeur associés par les locuteurs aux catégories d'objets sont inscrits au niveau du sens des mots désignant ces catégories :

«Le trait axiologique est une propriété sémantique de certaines unités lexicales.»

(Kerbrat-Orecchioni, 1980 : p. 79)

Tout sémantisme, selon l'approche de Kerbrat-Orecchioni, devient le réceptacle d'une axiologie qui se réalise dans le discours lorsque les formes linguistiques sont actualisées. L'axiologisation, de ce fait, participe directement à l'orientation argumentative du discours altermondialiste. On avance également l'hypothèse qu'elle contribuerait aussi au phénomène de persuasion discursive. Les énoncés ci-dessous attestent de ce procédé discursif :

(7) «La nécessité de lutter contre l'idée néolibérale qui voudrait que tous les États soient forcément corrompus, bureaucratiques, inefficaces ne rend que plus pressante la lutte qui doit être menée contre les déviations bureaucratiques, technocratiques et autoritaires des États. » (Texte 6) 
(8) «Appuyée sur les luttes pour la démocratisation, une réforme du système international peut être proposée. Elle comprend: la démocratisation du fonctionnement des institutions qui doivent mettre en œuvre la régulation internationale; la mise en place d'instances d'arbitrage et de recours efficaces; la priorité dans le système international de la lutte contre l'impunité; l'intégration effective dans le système direct des Nations Unies des institutions financières et commerciales internationales. » (Texte 10)

L'axiologisation concerne non seulement les valeurs axiologiques mais aussi les valeurs ontologiques, finalisantes et de jugement de vérité. Il semble que la persuasion discursive privilégie celles correspondant aux valeurs éthique et morale qui appartiennent à la catégorie des axiologiques. Ce qui attire notre attention dans les énoncés (7) et (8) se sont les syntagmes nominaux et verbaux suivants : «La nécessité de lutter contre l'idée néolibérale ", " la lutte qui doit être menée contre les déviations bureaucratiques ", "les luttes pour la démocratisation ", " une réforme du système international », « la lutte contre l'impunité » portent ces valeurs. Ce sont des segments répétés. Ils sont fréquemment employés et œuvrent à la conclusion argumentative des énoncés. Ce phénomène s'accentue dans le dernier énoncé ci-dessus où l'alternative à la politique des institutions internationales apparaît clairement: "l'intégration effective dans le système direct des Nations Unies des institutions financières et commerciales internationales ».

Les segments concernés par la lutte relèvent presque toutes de cette axiologisation (éthique, morale et pragmatique) dans l'intégralité du discours altermondialiste. Ce phénomène permet de baliser le discours altermondialiste d'expressions marquantes puisqu'elles possèdent la même forme et sont répétées régulièrement. Ces repères discursifs véhiculent donc une orientation argumentative. Cette dernière concourt à la persuasion discursive puisqu'elle soumet au destinataire des représentations autres du monde qui peuvent être partagées par les locuteurs. La persuasion est effective également parce que le mot « lutte » spécifie et systématise ces références sémantiques et cognitives.

Les problèmes et les solutions sont présentés en même temps dans ces expressions standardisées. Le destinataire peut se demander quel est alors le projet de l'altermondialisation. À cet effet, les organisations altermondialistes se sont fixées des objectifs pour un autre monde possible qui évolue selon les besoins et les priorités. CADTM fait circuler des mots pour y répondre et annonce ses intentions politiques. Elle adopte trois approches dans la construction du discours altermondialiste.

La première relève de la désignation de l'adversaire. Son objectif d'une altermondialisation concerne au premier abord la stigmatisation de la mondialisation (le néolibéralisme et la politique des institutions internationales). Elle est portée par des organisations variées mais d'obédience altermondialiste.

La deuxième approche est globale. Son objectif est de faire participer tout le monde (participation globale) contre les formes néolibérales de la mondialisation. Elle est menée par des militants altermondialistes de tous les pays. Les idées altermondialistes transcendent les frontières car la politique des institutions internationales s'appliquent presque sur l'ensemble des pays du globe.

La dernière approche concerne essentiellement l'altermondialisation elle-même. Son objectif se focalise sur la création d'un espace alternatif à la mondialisation en faisant 
converger les instances contestataires internationales vers un changement de modèle de société imposé et incertain.

Parmi ces trois approches, le discours altermondialiste fonctionne essentiellement sur les deux premières comme l'atteste, entre autres, la persuasion sémantique de ce discours. La construction de l'altermondialisation se fait par étapes et les approches ne correspondent pas à une démarche globale car elles répondent aux priorités du moment. Ce fait détermine le traitement sémantique du discours altermondialiste et influence les valeurs modales. Les valeurs éthique, morale et pragmatique sont fortement présentes et complètent les valeurs déontiques des mots car elles permettent d'orienter implicitement la conclusion et la réception des discours altermondialistes.

Nous avons analysé dans cette contribution des textes numériques appartenant à des typologies textuelles différentes et provenant de sources énonciatives différentes, afin de proposer une réflexion sur l'ethos collectif du mouvement altermondialiste. La présence de véritable polarisation entre un nous et un eux (l'adversaire) confère au mouvement altermondialiste un ethos montré combattant, qui s'oppose à un ethos dit qui joue sur le clivage entre une scénographie de combattant et une scénographie de pacifiste. La scénographie de lutte contre la mondialisation néolibérale se cantonne au champ sémantique de l'altérité et aux citations de militants et d'experts en économie. Si l'ethos dit reste en équilibre entre pacifisme et conflictualité, l'ethos montré reste résolument du côté du pacifisme et de l'inclusion: le groupe se veut solidaire à l'intérieur. L'ethos collectif renvoie donc à l'image avec laquelle les militants du mouvement peuvent s'identifier, avec sa part de différences (qui peut générer une certaine conflictualité) et sa part d'inclusion (nécessaire à la cohésion d'un mouvement). Ainsi, l'ethos collectif désigne l'ensemble des images de soi que l'orateur collectif projette dans son discours et qui sont à la base du pacte entre l'individu et l'instance collective dans laquelle il se reconnaît. L'ethos projeté est en premier lieu dit. Quant à l'ethos montré que projettent les altermondialistes, il est élaboré par le moyen du choix des pronoms (nous d'inclusion), celui des arguments et des procédés qu'ils utilisent pour retravailler leur ethos collectif.

\section{BIBLIOGRAPHIE}

AMOSSYRuth, «L'ethos au carrefour des disciplines : rhétorique, pragmatique, sociologie des champs ", dansAMOSSY RUTH, (dir.), Images de soi dans le discours, La construction de l'ethos, Genève,Delachaux et Niestlé,1999,pp. 127-154.

AMOSSY, R., La présentation de soi. Ethos et identité verbale, Paris, PUF, 2010.

BENVENISTE, É., Problèmes de linguistique générale, T1, Paris, Gallimard, 1966.

CHARAUDEAU, P., Le discours politique : les masques du pouvoir, Paris, Librairie Vuibert, 2005.

DECLERC, G., L'art d'argumenter. Structures rhétoriques et littéraires, Paris, Éditions Universitaires, 1993. 
GOLDER, C., « La production de discours argumentatifs : revue de questions », Revue française de pédagogie, $\mathrm{N}^{\circ}$ 4, Paris, 1996, pp. 119-134.

KERBRAT ORECCHIONI, C., La connotation, Lyon, PUL, 1977.

KERBRAT ORECCHIONI, C., Le discours polémique, Lyon, PUL, 1980.

ORKIBI, E., « Ethos collectif et Rhétorique de polarisation : le discours des étudiants en France pendant la guerre d'Algérie », Argumentation \& analyse du discours, $\mathrm{N}^{\circ} 1$, Paris, 2008,Disponible sur <http://journals.openedition.org/aad/438>(consulté le 14 février 2020).

ORKIBI, E., Les étudiants de France et la guerre d'Algérie. Identité et expression collective de l'UNEF (1954-1962), Paris, Syllepse, 2012.

\section{RÉSUMÉS}

Le mouvement altermondialiste fait partie de la constellation des formes de mobilisation citoyenne que semblent préférer les altermondialistes qui délaissent de plus en plus les formes de lutte traditionnelles. Nés vers la fin des années quatre-vingt-dix, les forums sociaux mondiaux sont aujourd'hui répandus dans le monde entier, notamment aux USA, dans les pays d'Amérique $\mathrm{du}$ Sud et en Afrique. Notre article va donc analyser la construction de l'image de soi qui s'est effectuée dans le discours altermondialiste et la façon dont elle s'est mise en place à travers une polarisation entre mondialisation et altermondialisation. En raison de la visée argumentative de cette construction, nous recourons à la notion d'ethos collectif, à savoir l'image de soi que le locuteur construit à travers son discours pour exercer une influence sur son auditoire.

The alterglobalist movement is part of the constellation of forms of citizen mobilization that today seem to be preferred by alterglobalists who are increasingly abandoning traditional forms of struggle. Born in the late nineties, the world social forums are today spread all over the world, especially in the USA, in the countries of South America and in Africa. Our article will therefore analyze the construction of the self-image that was carried out in the altermondialist discourse and the way in which it was established through a polarization between globalization and alterglobalization. Because of the argumentative aim of this construction, we resort to the notion of collective ethos, namely the self-image that the speaker builds through his speech to exert an influence on his audience.

\section{INDEX}

Mots-clés : ethos, forum, identité, autorité, référence

Keywords : ethos, forum, identity, authority, reference

\section{AUTEUR}

\section{AZZEDINE KADIR}

Université de Tizi-Ouzou, Algérie 